\title{
Reliability and Validity of the New Shooting Accuracy Measurement (SAM) System Software
}

\author{
Ugur Fidan $^{1}$ and Mehmet Yıldiz ${ }^{2}$ \\ 1. Department of Biomedical Engineering, Faculty of Sport Engineering, Afyon Kocatepe University, Afyonkarahisar 03200, Turkey \\ 2. School of Physical Education and Sports, Afyon Kocatepe Universit, Afyonkarahisar 03200, Turkey
}

\begin{abstract}
The aim of this study was to investigate the reliability and validity of the New Shooting Accuracy Measurement Software (SAMS). Thirty two male collegian soccer players performed three inner side kicking to the transparent goal construction. A High speed camera was placed at the back of the construction to determine where the ball contacted. Recorded video results were assessed with the SAMS and Kinovea software. To investigate test-retest reliability, three kicking results were examined twice by SAMS. Moreover kicking accuracy results obtained with SAMS were compared with results provided by Kinovea in order to investigate validity. Test-retest reproducibility of the new software was excellent, with Concordance Correlation Coefficient for distance to target $(0.99,0.99$ and 0.99 respectively) and for angular degree of the ball $(0.97,0.99$ and 0.99 respectively), low Coefficients of Variation (between 2.10 to 6.33 for distance to target and 2.40 to 2.69 for angular degree) and random error (between \pm 0.55 to \pm 3.44 for distance to target and \pm 0.63 to \pm 2.75 for angular degree). Constant error (between 0.44 to 1.28 for distance to target and -1.16 to \pm -2.51 for angular degree) and proportional error (between 0.97 to 0.98 for distance to target and 1.00 to 1.01 for angular degree) were very low for validity. In conclusion, the SAMS represents a valid and reliable instrument to measure accuracy of shooting to target.
\end{abstract}

Key words: Shooting, kicking, accuracy, measurement, distance.

\section{Introduction}

It is well known that soccer is the most popular sport in the world [1, 2] played by almost 265 million participants [3] who are men, women, children and adults with different levels of expertise. Soccer performance depends on a lot of factors such as technical, tactical, physical, physiological and mental areas [4] in which mobilization components such as dribbling, force movement timing to trap an approaching ball, direction control in juggling, and accuracy and power to kick a moving or a static ball are considered as the mastering tasks [5]. It is generally assured that kicking is the main effective action during the game in all these mastering tasks [6]. Because the more kicks directed to the goal, the better chances to score, and as a result coming closer to win. Therefore, kicking accuracy is an essential task of soccer

Corresponding author: Mehmet Y1ldiz, Ph.D., Dr., assistant professor, research field: exercise physiology and kinesiology. performance, and the improvement of kicking technique is one of the most important aims of soccer training for younger players [7]. There are a lot of studies focusing on this topic in the related literature [6, 8-14].

Accuracy can be defined as the ability to kick a ball to a special area [7] and tested by many different ways (Hitting a target, number of shots on goal or passing a ball between two markers) $[7,15,16]$. Nevertheless, all of these measurements have shortcomings. For example, the number of shots on goal in a per game may depend on a player's, goalkeeper's or opposite player's positions and skills. Furthermore, it is not necessary to kick the ball accurately to the goal. In hitting a target or passing the ball between two markers, calculation is not a factor in the magnitude of error when the target is missed. Moreover, in some studies, shooting accuracy was determined by measuring distance from ball contact point to target area $[17,18$, 19] using camera record and motion analyze software. 
This system seems more valid than other methods. However, disadvantage of this software is that it is necessary to use slow motion mode, continue frame by frame to detect the exact frame in which ball contacts the construction and analyze distance to target and angular degree of the ball to target area. This situation causes to considerable loss of time. To solve this problem, we have developed a new software in which whenever the ball contacts to the goal construction, the program catches the frames and measures distances and angular degrees automatically. Therefore the aim of this study was to investigate the reliability and validity of the New Shooting Accuracy Measurement (SAM) System Software.

\section{Methods}

\subsection{Participants}

Thirty-two male collegian soccer players (age 21.08 \pm 2.04 ; height $175.65 \pm 5.53$; body mass $69.79 \pm 6.96$ ) volunteered to participate in this study which was approved by the ethics committee in accordance with the Declaration of Helsinki. All subjects gave their written consents for participation in the study.

\subsection{Procedures}

The study was conducted on a $30 \times 40 \mathrm{~m}$ indoor testing area. After a full explanation of the experimental procedures, the subjects completed a standardized warm-up which consisted of running at a freely chosen speed (5-10 minutes) and freely chosen stretching of lower extremity muscles. Then they performed five inner side foot kicks to the designed goal construction for familiarization with the testing area. All kicks were performed with a stationary ball from a distance of $6 \mathrm{~m}$ to the target goal construction. Then participants were asked to perform three inside kicks to the goal construction which covered with a spanned and transparent sheet and have bull's eye target ( $1 \mathrm{~m}$ height) in the middle of a $3 \times 2 \mathrm{~m}$ [17]. A high speed camera (GoPro HERO4 black, USA) was placed at the back of the construction to determine the point where the ball contacted. The recorded results were assessed by the SAMS and Kinovea (Japan) software to determine the distance to the target and angular degrees. Two separate studies were conducted to determine the reliability and validity of the new software. In the first study, three kicking results were examined twice with SAMS at different times to determine the test-retest reliability. In the second study, kicking accuracy results obtained by the new software were compared with results provided by Kinovea software program to investigate concurrent validity. Kinovea program is highly used by sport scientists in motion analyzes studies [20-22]. Therefore, it was selected as a gold standard to evaluate the validity.

\subsection{The New Software}

This software was written in the MATLAB Program. The Screenshot of the new software is seen in Fig. 1. It can be used only with the integrated system of Sterzing et al. [17]. First, a video capturing kicking performance to the target is loaded. Then the midpoint of the target, $50 \mathrm{~cm} \mathrm{x}$ and $50 \mathrm{~cm}$ y plane is marked with little square on screen for calibration. After that, whenever the ball contacts to the goal construction, the program catches these frames and measures distances and angular degrees automatically. The distance to target and angular degrees appear on screen numerically and graphically.

\subsection{Statistical Analyze}

Statistical analysis was conducted using SPSS 18.0 (SPSS Inc., Chicago, IL, USA). The results were expressed as mean $\pm \mathrm{SD}$. Student's t-tests were used to detect any difference between software (validity) and test sessions (reliability). Reliability of the New Software was examined using the Concordance Correlation Coefficient (CCC), Coefficients of Variation (CVs), and Bland-Altman systematic bias \pm random error. The Deming Regression Method was used to determine validity of the SAMS. After determining the compatibility in Bland-Altman plot graphic, the 


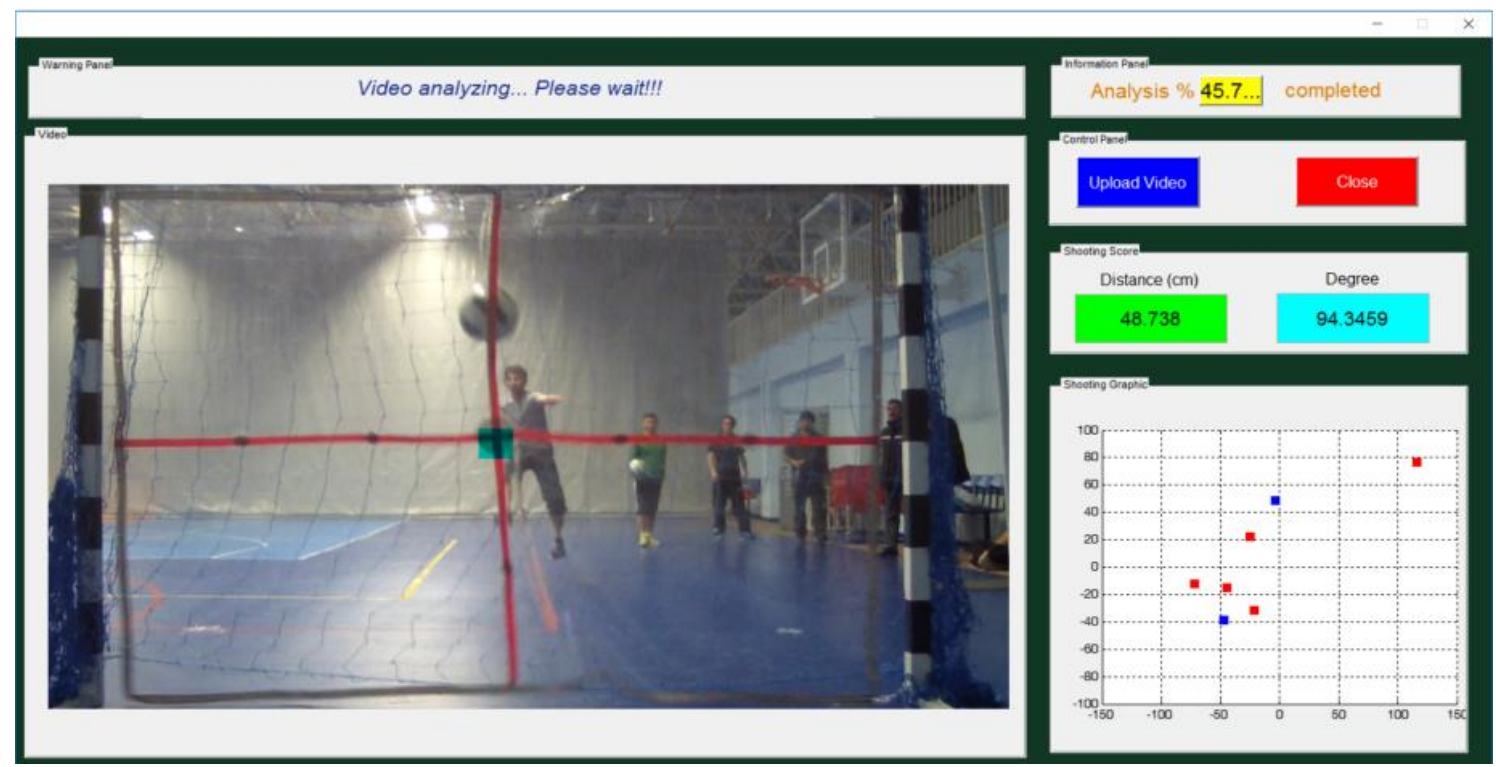

Fig. 1 The Screenshot of the new software.

Table 1 Test-retest reliability of the SAMS software for distance to the target and the angular degree of the ball of the three kicks.

\begin{tabular}{|c|c|c|c|c|c|c|}
\hline & & $\bar{x} \pm s \mathrm{~d}$ & $\mathrm{CCC}$ & $\begin{array}{l}\text { CV } \\
(\%)\end{array}$ & Syste & Random error \\
\hline \multirow{4}{*}{ Frist kick } & Test (Distance) $(\mathrm{cm})$ & $55.78 \pm 27.48$ & \multirow{2}{*}{0.99} & \multirow{2}{*}{2.44} & \multirow{2}{*}{-1.19} & \multirow{2}{*}{ \pm 0.83} \\
\hline & Retest (Distance) $(\mathrm{cm})$ & $56.06 \pm 27.04$ & & & & \\
\hline & Test (Degree) $\left(^{\circ}\right)$ & $181.7 \pm 91.92$ & \multirow{2}{*}{0.97} & \multirow{2}{*}{2.40} & \multirow{2}{*}{0.50} & \multirow{2}{*}{ \pm 0.63} \\
\hline & Retest (Degree) $\left(^{\circ}\right)$ & $179.1 \pm 90.25$ & & & & \\
\hline \multirow{4}{*}{ Second kick } & Test (Distance) $(\mathrm{cm})$ & $42.62 \pm 25.37$ & \multirow{2}{*}{0.99} & \multirow{2}{*}{2.10} & \multirow{2}{*}{-0.49} & \multirow{2}{*}{ \pm 0.55} \\
\hline & Retest (Distance) $(\mathrm{cm})$ & $42.69 \pm 25.71$ & & & & \\
\hline & Test (Degree) $\left(^{\circ}\right)$ & $188.7 \pm 101.94$ & \multirow{2}{*}{0.99} & \multirow{2}{*}{2.63} & \multirow{2}{*}{3.90} & \multirow{2}{*}{ \pm 2.02} \\
\hline & Retest (Degree) $\left(^{\circ}\right)$ & $185.5 \pm 103.24$ & & & & \\
\hline \multirow{4}{*}{ Third kick } & Test (Distance) $(\mathrm{cm})$ & $46.38 \pm 28.43$ & \multirow{2}{*}{0.99} & \multirow{2}{*}{6.33} & \multirow{2}{*}{0.87} & \multirow{2}{*}{ \pm 3.44} \\
\hline & Retest (Distance) $(\mathrm{cm})$ & $46.16 \pm 27.99$ & & & & \\
\hline & Test (Degree) $\left(^{\circ}\right)$ & $171.8 \pm 89.95$ & \multirow{2}{*}{0.99} & \multirow{2}{*}{2.69} & \multirow{2}{*}{1.98} & \multirow{2}{*}{ \pm 2.75} \\
\hline & Retest (Degree) $\left(^{\circ}\right)$ & $170.3 \pm 90.20$ & & & & \\
\hline
\end{tabular}

$\mathrm{CCC}$, the Concordance Correlation Coefficient. $\bar{x}$, mean. sd, Standard deviation. CV, Coefficient variation.

Deming Regression method was applied to determine systematic bias (constant and proportional error). Statistical significance was set at $P<0.05$.

\section{Results}

Test-retest reliability of the SAMS software for distance to the target and the angular degree of the ball of the three kicks are shown in Table 1. Test-retest reproducibility of the new software was excellent, with CCC for distance to target $(0.99,0.99$ and 0.99 respectively) and for angular degree of the ball ( 0.97 ,
0.99 and 0.99 respectively), low CVs (between 2.10 to 6.33 for distance to target and 2.40 to 2.69 for angular degree) and random error (between \pm 0.55 to \pm 3.44 for distance to target and \pm 0.63 to \pm 2.75 for angular degree). Systematic bias was nonsignificant and very close to 0 .

The results of the Deming Regression Analysis comparing the SAMS and Kinovea software are shown in Table 2. Constant error (between 0.44 to 1.27) and proportional error (between 0.97 to 0.98 ) were very low in terms of the distance to target. Moreover, constant 
Table 2 Concurrent validity of the SAMS and KINOVEA software for distance to the target and the angular degree of the ball of the three kicks.

\begin{tabular}{|c|c|c|c|c|}
\hline & & $\bar{x} \pm s \mathrm{~d}$ & $\begin{array}{l}\text { Deming } \\
\beta_{0}(95 \% \mathrm{cl})\end{array}$ & $\begin{array}{l}\text { Deming } \\
\beta_{1}(95 \% \mathrm{cl})\end{array}$ \\
\hline \multirow{4}{*}{ First kick } & SAMS (Distance) (cm) & $55.78 \pm 27.48$ & \multirow{2}{*}{$1.28(-1.12$ to 3.68$)$} & \multirow{2}{*}{0.97 ( 0.94 to 1.03$)$} \\
\hline & Kinovea (Distance) $\mathrm{cm}$ & $56.47 \pm 26.63$ & & \\
\hline & SAMS (Degree) $\left({ }^{\circ}\right)$ & $181.7 \pm 91.92$ & \multirow{2}{*}{$-2.52(-6.19$ to 1.16$)$} & \multirow{2}{*}{1.01 ( 0.99 to 1.03$)$} \\
\hline & Kinovea (Degree) $\left(^{\circ}\right)$ & $181.0 \pm 92.81$ & & \\
\hline \multirow{4}{*}{ Second kick } & SAMS (Distance) $(\mathrm{cm})$ & $42.62 \pm 25.37$ & \multirow{2}{*}{$0.57(-0.62$ to 1.77$)$} & \multirow{2}{*}{0.98 ( 0.96 to 1.02$)$} \\
\hline & Kinovea (Distance) $(\mathrm{cm})$ & $42.44 \pm 24.92$ & & \\
\hline & SAMS (Degree) $\left(^{\circ}\right)$ & $188.7 \pm 101.94$ & \multirow{2}{*}{$-0.16(-3.68$ to 2.48$)$} & \multirow{2}{*}{$1.01(0.99$ to 1.03$)$} \\
\hline & Kinovea (Degree) $\left(^{\circ}\right)$ & $186.8 \pm 100.28$ & & \\
\hline \multirow{4}{*}{ Third kick } & SAMS (Distance), $(\mathrm{cm})$ & $46.38 \pm 28.43$ & \multirow{2}{*}{$0.44(-0.58$ to 1.46$)$} & \multirow{2}{*}{$0.98(0.95$ to 1.00$)$} \\
\hline & Kinovea (Distance) $(\mathrm{cm})$ & $45.81 \pm 27.81$ & & \\
\hline & SAMS (Degree) $\left(^{\circ}\right)$ & $171.8 \pm 89.95$ & \multirow{2}{*}{$-1.22(-5.13$ to 2.69} & \multirow{2}{*}{$1.00(0.98$ to 1.02$)$} \\
\hline & Kinovea (Degree) $\left(^{\circ}\right)$ & $171.1 \pm 90.18$ & & \\
\hline
\end{tabular}

$ß_{0}$, intercept. $\aleph_{1}$, slope. cl, Confidence Interval. $\bar{x}$, mean. sd, Standard Deviation.

error (between -1.16 to -2.51) and proportional error (between 1.00 to 1.01 ) were very low with regards to angular degree of the ball to target too. Because of including confidence interval " 0 " for constant error and " 1 " for proportional error, it was not seen a systematic bias.

\section{Discussions and Conclusions}

The new designed shooting accuracy measurement (SAM) system software was used to evaluate kicking accuracy (distance and angular degree of the ball to target). The new system software represented a valid and reliable instrument to measure accuracy of shooting by measuring distance to target and angular degree of the ball.

In the literature, many different ways have been used to determine kicking or shooting accuracy. Hitting a target, number of shots on goal and passing the ball between two markers are common methods [7, 14-16]. However, each of them had shortcomings as mentioned above. For example, the number of shots on goal in a per game may depend on a player's, goalkeeper's or opposite player's positions and skills. Furthermore, it is not necessary to kick the ball accurately to the goal. In hitting a target or passing the ball between two markers, calculation is not a factor in the magnitude of error when the target is missed. To solve these problems, Finnof et al. [7] offered a valid and reliable method to measure kicking velocity for soccer players. In their study, shooting accuracy was determined by measuring the distance from the ball contact point to the special target area [7] using plywood surface covered by sheeds carbon papers with carbon side in contact with the white paper. This method provides information on the degree of accuracy as opposed to the ability to hit simply or miss the target. However, this method does not seem appropriate for practical use. Similar to Finnof et al. [7], Bjelica et al. [13, 14] used a special target area in which the centre of the target was marked with cross lines which divided the target to four equal rectangles. From the central point, many concentric circles were drawn, and the first one had the same diameter as a standard ball $(22.1 \mathrm{~cm})$. All the other circles were outlined by their mutual space between a size of a standard ball diameter. The central circle brought 17 points which were the maximal number of points for one shoot whereas peripheral circles on the left and right brought one point. It means that every shoot closer to centre brought a larger number of points while every failure was identified as zero points. This method seems more valid but it is not practical. Because the tester has to 
catch the contact point of the ball using his/her eyes. Thus this is a subjective measurement when ball speed increases. This method can be applicable only in using a camera system to catch the contact point of the ball. Similar to this method, Sterzing et al. [17] used a camera and motion analysis software program to measure the ball's contact area covered with a slightly spanned and transparent sheet. In this system, a camera is used behind the target area to catch the ball contact point. This system seems more valid than the other methods. In another study, Scurr and Hall [19] used camera record and motion analyses program to measure penalty accuracy. However, in this system and software, it is necessary to use slow motion mode, continue frame by frame, find the frame in which a ball contact the construction, and analyze distance to target. This situation leads to considerable loss of time. Because, the software used in the sport field to analyze video based motion is designed for a wide use. To solve this problem in the new software program in which whenever the ball contacts to the goal construction, the program catches these frames, measures distances and angular degrees automatically. Main advantage of this software program is to use time effectively. This software measures not only the distance to the target but also angular degrees of points to the target. Moreover, players' results can be compared with each other and technical development can be observed over time. Tester can change the target point place (bull's eye) allowing players to practice kicking to specific spots on the goal. Using a high speed camera is recommended for obtaining more correct results. Because when speed of the ball is increased, the software may not catch the real frame in which ball contacts to the target construction in a low speed camera records.

In conclusion, over the past decade, increasing capacity of digital technology to collect, manage and organize video images has made it possible to enhance existing sport specific analytical procedures [23]. For example, human body models that were initially described in 2D have recently evolved into highly articulated 3D models [24]. Moreover, a variety of systems and methods have been employed to analyze the motion of athletes during sports where the movements vary in duration, field position and surface, speed and direction technique and tactics [23]. Technological advances have included the introduction of increasingly sophisticated motion analysis systems that are now being used in elite soccer not only to determine physical demands of match-play but also to analyze a variety of techniques. Video cameras linked with computers and automated analysis software provide a more sophisticated approach to motion analysis in order to have a more accurate result [23]. Therefore, specific software programs have become a necessity for more accurate results and saving time. The new software (SAMS) represents a valid, reliable and effective instrument as for time to measure the accuracy of shooting to a target.

\section{Reference}

[1] Bangsbo, J. 1993. "The Physiology of Soccer with Special Reference to Intense Intermittent Exercise." Acta physiologica Scandinavica. Supplementum 619 (1): 1-155.

[2] Lees, A., and Nolan, L. 1998. "The Biomechanics of Soccer: A Review." Journal of Sports Sciences 16 (3): 211-34.

[3] Kunz, M. 2007. "265 Million Playing Football.” FIFA Magazine, 10-5. Accessed June 26, 2016. http://www.fifa.com/mm/document/fifafacts/bcoffsurv/e maga_9384_10704.pdf

[4] Stølen, T., Chamari, K., Castagna, C., and Wisløff, U. 2005. "Physiology of Soccer." Sports Medicine 35 (6): 501-36.

[5] Teixeira, L. A., Oliveira, D. L., Romano, R. G., and Correa, S. C. 2011. "Leg Preference and Interlateral Asymmetry of Balance Stability in Soccer Players.” Research Quartely Exercises in Sport 82 (1): 21-7.

[6] Kellis, E. L., and Katis, A. 2007. "Biomechanical Characteristics and Determinants of Instep Soccer Kick." Journal of Sport Science and Medicine 6 (2): 154-65.

[7] Finnoff, J. T., Newcomer, K., and Laskowski, E. R. 2002. "A Valid and Reliable Method for Measuring the Kicking Accuracy of Soccer Players." Journal of Science and Medicine in Sport 5 (4): 348-53.

[8] Tracey, S. Y., Anderson, D. I., Hamel, K. A., Gorelick, M. L., Wallace, S. A., and Sidaway, B. 2012. "Kicking 
Performance in Relation to Balance Ability over the Support Leg." Human Movement Science 31 (6): 1615-23.

[9] Barone, R., Macaluso, F., Traina, M., Leonardi, V., Farina, F., and Di Felice, V. 2011. "Soccer Players Have a Better Standing Balance in Nondominant One-Legged Stance.” Journal of Sports Medicine 2 (1): 1-6.

[10] Lees, A., Asai, T., Andersen, T. B., Nunome, H., and Sterzing, T. 2010. "The Biomechanics of Kicking in Soccer: A Review." Journal of Sports Sciences 28 (8): 805-17.

[11] Nunome, H., Ikegami, Y., Kozakai, R., Apriantono, T., and Sano, S. 2006. "Segmental Dynamics of Soccer Instep Kicking with the Preferred and non-Preferred Leg." Journal of Sports Sciences 24 (5): 529-41.

[12] Dörge, H. C., Andersen, T. B., SØrensen, H., and Simonsen, E. B. 2002. "Biomechanical Differences in Soccer Kicking with the Preferred and the non-Preferred Leg." Journal of Sport Science 20 (4): 293-9.

[13] Bjelica, D., Georgiev, G., and Popović, S. 2011. "Comparison of Instep Kicking by non-Preferred Leg among Various States and Intensities in Young Football Players." Acta Kinesiologica 5 (1): 79-82.

[14] Bjelica, D., Popovic, S., and Petkovic, J. 2013. "Comparison of Instep Kicking Between Preferred and Non-Preferred Leg in Young Football Players." Montenegrin Journal of Sports Science and Medicine 2 (1): 5-10.

[15] Carey, D. P., Smith, G., Smith, D. T., Shepherd, J. W., Skriver, J., Ord, L., and Rutland, A. 2001. "Footedness in World Soccer: An Analysis of France'98." Journal of Sports Sciences 19 (11): 855-64.

[16] McLean, B. D., and Tumilty, D. M. 1993. "Left-right
Asymmetry in Two Types of Soccer Kick." British Journal of Sports Medicine 27 (4): 260-2.

[17] Sterzing, T., Lange, J. S., Wächtler, T., Müller, C., and Milani, T. L. 2009. "Velocity and Accuracy as Performance Criteria for Three Different Soccer Kicking Techniques." In ISBS-Conference Proceedings Archive.

[18] Scurr, J., and Hall, B. 2009. "The Effects of Approach Angle on Penalty Kicking Accuracy and Kick Kinematics with Recreational Soccer Players." Journal of Sports Science and Medicine 8 (3): 230-4.

[19] Majelan, A. S., Rahmani-Nia, F., Norasteh, A. A., and Damirchi, A. 2011. "The Effects of Approach Angle and Target Position on Instep Kicking Accuracy and Ball Speed with Skilled Soccer Players." Sport SPA 8 (2): 35-9.

[20] McGinnis, P. M. 2013. Biomechanics of Sport and Exercise. Leeds, United Kingdom: Human Kinetics.

[21] Silva, M. G., and Hirata, T. 2013. "Kicking Performance for Soccer Players with Biomechanical Instrumentation." Presented at the 22nd International Congress of Mechanical Engineering, SP, Brazil.

[22] Balsalobre-Fernández, C., Tejero-González, C. M., del Campo-Vecino, J., and Bavaresco, N. 2014. "The Concurrent Validity and Reliability of a Low-cost, High-speed Camera-based Method for Measuring the Flight Time of Vertical Jumps." The Journal of Strength \& Conditioning Research 28 (2): 528-33.

[23] Barris, S., and Button, C. 2008. "A Review of Vision-Based Motion Analysis in Sport." Sports Medicine 38 (12): 1025-43.

[24] Poppe, R. 2007. "Vision-based Human Motion Analysis: An Overview." Computer Vision and Image Understanding 108 (1): 4-18. 